\title{
Clasificador de atención de la inmediatez en el área de servicios de urgencias Médicas
}

\section{Classifier of immediate attention in the area of medical emergency services}

LEON-SOSA, Sandra Elizabeth†*, HERNÁNDEZ-BÁEZ, Irma Yazmín, SOLANO-TAPIA, Jaime Alberto y MORALES-MORALES, Cornelio

Universidad Politécnica del Estado de Morelos

ID $1^{\text {er }}$ Autor: Sandra Elizabeth, León-Sosa / ORC ID: 0000-0002-6047-4713, CVU CONACYT ID: 648491

ID $1^{\text {er }}$ Coautor: Irma Yazmín, Hernández-Báez / ORC ID: 0000-0002-3078-9618, CVU CONACYT ID: 212658

ID $2^{\text {do }}$ Coautor: Jaime Alberto, Solano-Tapia / ORC ID: 0000-0001-8490-5867

ID $3^{\text {er }}$ Coautor: Cornelio, Morales-Morales / ORC ID: 0000-0002-3470-4626, CVU CONACYT ID: 172942

DOI: $10.35429 / J C T .2019 .10 .3 .1 .8$

Recibido 22 de Julio, 2019, Aceptado, 24 de Agosto, 2019

Resumen

En México y Latinoamérica se ha hecho necesario entrenar al personal de urgencias para efectuar la primera etapa del triage a fin de optimizar los recursos humanos disponibles en los hospitales. Por lo tanto, la presente investigación desarrolla una aplicación móvil que permita registrar los síntomas del paciente al acudir al área de servicios de urgencias y puedan ser clasificados mediante una Red bayesiana en un entorno de aprendizaje supervisado que permita al personal médico del hospital determinar el procedimiento que debe de aplicar al paciente. Los datos recopilados de la aplicación móvil son almacenados en un sistema web, por lo tanto, los síntomas son utilizados como atributos para ser clasificados y realizar un pronóstico de la posible enfermedad, que determina la prioridad de la atención médica utilizando los colores de las guías prácticas de las clínicas de salud, por consiguiente, se muestra el mensaje indicando el color, el tiempo de respuesta y un posible diagnóstico de la enfermedad.

Triage, Sistema, Clasificador

\begin{abstract}
In Mexico and Latin America it has become necessary to train emergency personnel to carry out the first stage of triage in order to optimize the human resources available in hospitals. Therefore, this research develops a mobile application that allows the patient's symptoms to be recorded when they go to the emergency services area and can be classified by a Bayesian network in a supervised learning environment that allows the hospital's medical staff to determine the procedure which must apply to the patient. The data collected from the mobile application is stored in a web system, therefore the symptoms are used as attributes to be classified and to make a prognosis of the possible disease, which determines the priority of the medical care using the colors of the practical guides of the health clinics, therefore the message is displayed indicating the color, the response time and a possible diagnosis of the disease
\end{abstract}

Triage, System, Classifier

Citación: LEON-SOSA, Sandra Elizabeth, HERNÁNDEZ-BÁEZ, Irma Yazmín, SOLANO-TAPIA, Jaime Alberto y MORALES-MORALES, Cornelio. Clasificador de atención de la inmediatez en el área de servicios de urgencias Médicas. Revista de Tecnología Informática. 2019 3-10: 1-8 


\section{Introducción}

Los servicios de urgencias se ha convertido en una barrera que impide a la población recibir una atención médica de urgencias oportuna, los pacientes enfrentan tiempos de espera prolongados para ser admitidos y tratados en el servicio; para ser ingresados a una cama de hospitalización. El área de servicios de urgencias médicas es parte importante de un sistema de salud, por la cantidad de pacientes que acuden a solicitar un servicio.

Aproximadamente $15 \%$ de las atenciones otorgadas en el Instituto Mexicano del Seguro Social se ofrece en el área de Urgencias. Los principales motivos fueron las infecciones respiratorias agudas $(19.4 \%)$, los traumatismos y envenenamientos $(18.8 \%)$ y las enfermedades diarreicas (8\%). El $21 \%$ de las defunciones en la institución ocurre en el área de urgencias, y la diabetes mellitus y las enfermedades cardiovasculares figuran entre las principales causas de muerte, sobre todo en la población adulta y adulta mayor (Cantón, 2006).

El proceso de clasificación de los pacientes que acuden a los servicios de urgencias hospitalarios se ha convertido en una necesidad debido a la gran demanda de atención y a la saturación que, en ocasiones, sufren dichos servicios. Es el área con mayor volumen de pacientes, de entre los cuales algunos padecen urgencias reales y otros no; sin embargo, todos exigen una atención rápida y de calidad. A partir de ahí surgen las evaluaciones que realiza la población a un hospital y su personal, así como la satisfacción que manifiesta de la atención recibida (Hernández \& León, 2012).

El objetivo de la presente investigación es desarrollar una aplicación móvil que permita al paciente registrar los síntomas al trasladarse a los servicios de urgencias, los datos son almacenados en un sistema web permitiendo clasificar el nivel de urgencia, por consiguiente, al ingresar a los servicios de urgencias agilice el acceso al Hospital para la atención médica. El sistema brindará apoyo al personal médico permitiendo observar los síntomas y el diagnóstico emitido por el sistema clasificando el nivel de urgencia de acuerdo a los colores de las guías prácticas de las clínicas de salud, teniendo un panorama de la posible enfermedad del paciente.
Se utiliza el clasificador de Naïve bayes para predecir la posible enfermedad del paciente, como el nivel de urgencia, mostrando un reporte al personal médico. Sirviendo como una herramienta de apoyo, que permita agilizar la recepción del paciente al momento de recibirlo y poder ofrecer la atención médica oportuna.

\section{Estado del Arte}

Benavente (2014) expone que las prestaciones en los servicios de urgencia, han creado la necesidad de introducir en dichos servicios un sistema de filtro que gestione la afluencia de los usuarios y que seleccione dentro de los servicios con el fin de tutelar aquellos pacientes que presenten patologías más graves, de tal manera que la atención a estos no se demore. Estos factores obligan a establecer sistemas de triage

En México se maneja el triage modificado del canadiense, en el Instituto Mexicano del Seguro Social, se utilizan los colores que se visualizan en la tabla 1, las cuales aparecen en las guías de práctica clínica y tiene como objetivo optimizar la atención del paciente.

\begin{tabular}{|c|c|c|c|}
\hline Nivel & Color & Significado & $\begin{array}{l}\text { Tiempo máximo } \\
\text { de atención }\end{array}$ \\
\hline I & Rojo & $\begin{array}{l}\text { Reanimación } \\
\text { Inmediata }\end{array}$ & Inmediatamente \\
\hline II & Anaranjado & Emergencia & $\begin{array}{l}\text { Menor a } 10 \\
\text { minutos }\end{array}$ \\
\hline III & Amarillo & Urgencia & $\begin{array}{l}\text { Menor a } 30 \\
\text { minutos }\end{array}$ \\
\hline IV & Verde & Poco urgente & $\begin{array}{l}\text { menos de } 60 \\
\text { minutos }\end{array}$ \\
\hline $\mathbf{V}$ & Azul & Sin urgencia & $\begin{array}{l}\text { Menor de } 240 \\
\text { minutos }\end{array}$ \\
\hline
\end{tabular}

Tabla 1 Clasificación triage del sistema CAST (Triage del departamento de Canadá). Fuente. (MA, AE, I, \& A, 2017)

Los sistemas basados en el conocimiento para ayudar a la toma de decisiones en la clasificación generalmente tratan de cubrir un dominio mucho más amplio, pero pueden usar un conjunto más pequeño de variables debido a restricciones de tiempo, muchas de ellas subjetivas, por lo que es difícil construir modelos precisos (Abad-Grau, Ierache, \& Cervino, 2008).

La afluencia de pacientes en el servicio de Urgencias es uno de los grandes problemas a los que se enfrentan los sistemas hospitalarios actualmente al acarrear problemas de sobrecarga y colapso. 
Por ello, se hace imprescindible para los profesionales de salud de estos servicios contar con un sistema de triage que priorice la atención de los pacientes y permita gestionar el riesgo clínico para manejar de forma adecuada y segura los flujos de pacientes (Arcos, 2017)

Las redes bayesianas se basan en los fundamentos de la teoría de la probabilidad y permiten combinar el juicio del experto con las fuentes de datos disponibles, y realizar inferencia entre cualquier subconjunto de variables.

Aunque tienen un amplio uso en el ámbito médico, en los sistemas de triage se han empezado a aplicar. Existen numerosos ejemplos en los que se muestran los buenos resultados que la redes bayesianas están teniendo en el diagnóstico médico (Abad-Grau, Ierache, \& Cervino, 2008).

Un clasificador bayesiano puede definirse para el triage hospitalario si la clase es el nivel de triage y las variables de entradas aquella información que deba tenerse en cuanta para inferir la clase. Uno de los problemas que han sido señalados por el personal de los servicios de urgencia como inconvenientes de los sistemas computacionales de triage es su limitación en cuanto a las variables que tienen en cuenta para determinar el nivel de triage (Seixas, Zadrozny, Laks, Conci, \& Saade, 2014)

Los modelos bayesianos primordialmente incorporan conocimiento previo para poder estimar modelos útiles dentro de un espacio muestral y de este modo poder estimar parámetros que provengan de la experiencia o de una teoría probabilística. (Páez, Lozano, \& Davila, 2011)

Las Naïve Bayes son uno de los modelos más simples y más utilizados, en estos modelos la hipótesis que se maneja es que los atributos son condicionalmente independientes de la variable a clasificar (Torra, 2017).

Rivera-Rodríguez (2012) expone el trabajo de investigación de un sistema móvil de tele asistencia médica para apoyar la atención de casos de emergencia, utilizando servicios de transmisión de signos vitales y videoconferencia en tiempo real.

\section{Metodología}

Para la selección de datos utilizados se utilizó la metodología KDD (Knowledge Discovery in Discovery) es básicamente un proceso automático en el que se combinan descubrimiento y análisis. El proceso consiste en extraer patrones en forma de reglas o funciones, a partir de los datos, para que el usuario los analice (Timarán-Pereira, Hernández-Arteaga, Caicedo-Zambrano, Hidalgo-Troya, \& Alvarado- Pérez, 2016).

Hernández J., Ramírez M. \& Ferri C. (2005) indica que es un proceso iterativo e interactivo organizado en cinco fases. Dentro de las fases se encuentran la integración de datos, la preparación de los datos y las técnicas de minería de datos. (J., M., \& C., 2004). La Figura 1, indica las etapas de la extracción y descubrimiento de conocimiento.

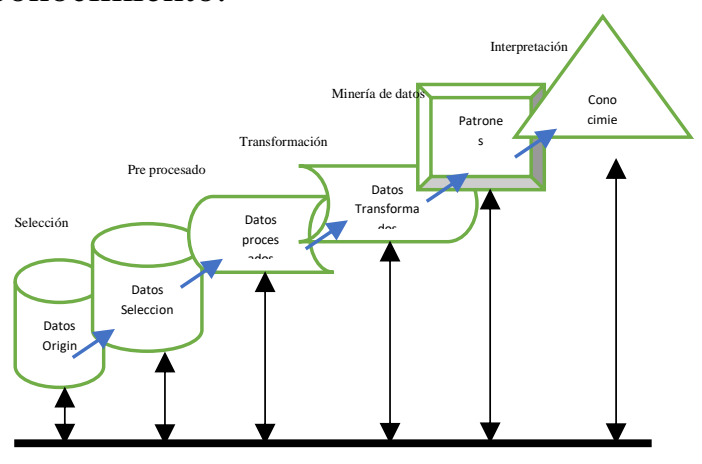

Figura 1 Etapas para el proceso de extracción de conocimiento

Fuente: (Morin \& Cervantes, 2018)

\section{A. Integración de datos}

Para la etapa de los datos recopilados se obtuvieron de doctores que residen en la Ciudad de Cuernavaca Morelos, de pacientes que acudieron a cita en el año del 2018, donde se obtuvieron un total de 250 registros que acudieron a servicios de urgencias.

\section{B. Preparación de datos}

La información se encuentra en un archivo en Excel, se procedió a realizar una limpieza de datos para ser preparados para el entrenamiento del algoritmo, por lo que se procedió realizar la limpieza de los mismos, con el objetivo de eliminar valores erróneos o desconocidos. Se utilizaron 13 atributos para realizar el diagnóstico de la enfermedad. 
Por mencionar algunos son: Resfriado, Gripa, Problemas del corazón, Infección estomacal, neumonía y Anginas por mencionar algunos.

\section{Algoritmo Propuesto}

El cálculo de probabilidades condicionales inversas mediante el teorema de Bayes es fundamental en las aplicaciones de la Estadística, porque permite incorporar cambios en nuestro grado de creencia sobre los sucesos aleatorios, a medida que adquirimos nueva información.

Este tipo de razonamiento es muy importante en tareas profesionales, como el diagnóstico, evaluación, toma de decisiones y aplicación de la inferencia estadística en la investigación empírica (Fuente, 2006).

Para el desarrollo del sistema se utiliza el razonamiento probabilístico, para obtener la clasificación de la urgencia y el posible diagnóstico de la enfermedad del paciente. Lo cual se basó en el Teorema de Bayes, para lo cual se acudió a los Médicos utilizando pruebas de diagnósticos diariamente para decidir el tipo de enfermedad de los pacientes.

El teorema de Bayes, representa un método estadístico para evaluar nueva información y tener una revisión de las estimaciones anteriores.

Sea $\{A 1, A 2, \ldots A i \ldots A n\}$ un conjunto de sucesos mutuamente excluyentes y cuya unión es el total o sea 1, y tales que la probabilidad de cada uno de ellos es distinta de cero.

Sea B un suceso cualquiera del que se conocen las probabilidades condicionales $\mathrm{P}$ $(\mathrm{B} / \mathrm{Ai})$. Entonces la probabilidad $\mathrm{P}(\mathrm{Ai} / \mathrm{B})$ viene dada por la expresión (Regalado, 2009):

$$
P(A i \mid B)=\frac{P(B \mid A i) P(A i)}{\sum_{j=1}^{n} P(B \mid A j) P(A j)}
$$

- $\quad$ P(Ai) son las probabilidades a priori.

- $\quad$ P(B/Ai) es la probabilidad de B en la hipótesis Ai.

- $\quad \mathrm{P}(\mathrm{Ai} / \mathrm{B})$ son las probabilidades a posteriori.
Para el entrenamiento de la red se utilizó un clasificador probabilístico fundamentado en la teoría de Bayes, para entrenar la red Naïve Bayes se utilizaron los síntomas del paciente para determinar el tipo de urgencias, son utilizadas 4 variables para estimar los parámetros necesarios para la clasificación, indicando el color de la urgencia y diagnosticando la posible enfermedad.

Para involucrar el algoritmo con el sistema, se utilizó el software Weka por sus siglas en inglés (Waikato Enviroment for Knowledge Analysis) el cual está diseñado para el análisis de datos.

Para la prueba y validación de minería de datos se realizó el proceso de evaluar datos reales, se coloca el proceso con una serie de atributos que son etiquetados, en la tabla $X$ se coloca un conjunto de 4 ejemplos, con 3 atributos cada uno y dos posibles clases Tabla 2.

\begin{tabular}{|c|c|c|c|l|}
\hline Ejemplos & Atr.1 & Atr. 2 & Atr.3 & Clase \\
\hline $\mathrm{x} 1$ & 1 & 2 & 1 & Positiva \\
\hline $\mathrm{x} 2$ & 2 & 2 & 2 & Positiva \\
\hline $\mathrm{x} 3$ & 1 & 1 & 2 & Negativa \\
\hline $\mathrm{x} 4$ & 2 & 1 & 2 & Negativa \\
\hline
\end{tabular}

Tabla 2 Clasificador de datos

Fuente: Elaboración Propia

Se establecen las probabilidades:

$\mathrm{P}($ positivo $)=0.5$

$\mathrm{P}($ negativo $)=0.5$

Se generan nuevas tablas de acuerdo a las clases y se hace un recuento de los valores Tabla 3.

\begin{tabular}{|c|c|c|}
\hline Positivo & \multicolumn{1}{|c|}{ Valor1 } & Valor2 \\
\hline Atr.1 & 1 & 1 \\
\hline Atr.1 & 0 & 2 \\
\hline Atr.3 & 1 & 1 \\
\hline
\end{tabular}

\begin{tabular}{|c|c|c|}
\hline Negativo & Valor1 & Valor2 \\
\hline Atr.1 & 1 & 1 \\
\hline Atr.1 & 2 & 0 \\
\hline Atr.3 & 0 & 2 \\
\hline
\end{tabular}

Tabla 3 Tabla de acuerdo de clases

Fuente: Elaboración Propia

Se suma una unidad a cada valor de las tablas, Tabla 4. 


\begin{tabular}{|c|c|c|}
\hline Positivo & Valor1 & Valor 2 \\
\hline Atr.1 & 2 & 2 \\
\hline Atr.1 & 2 & 3 \\
\hline Atr.3 & 2 & 2 \\
\hline
\end{tabular}

\begin{tabular}{|c|c|c|}
\hline Negativo & Valor1 & Valor2 \\
\hline Atr.1 & 2 & 2 \\
\hline Atr.1 & 3 & 1 \\
\hline Atr.3 & 1 & 3 \\
\hline
\end{tabular}

Tabla 4 Tablas con un valor agregado Fuente Elaboración Propia

Se normalizan todos los valores de las tablas del siguiente modo. Cada celda se divide por la suma de los valores de la fila. Por ejemplo, el valor: "valor 1" del atributo: "atr. 2" en la tabla 5 de positivo se actualiza con: $1 /(1+3)=0,25$

\begin{tabular}{|c|c|c|}
\hline Positivo & Valor1 & Valor2 \\
\hline Atr.1 & 0.5 & 0.5 \\
\hline Atr.1 & 0.25 & 0.75 \\
\hline Atr.3 & 0.5 & 0.5 \\
\hline
\end{tabular}

\begin{tabular}{|c|c|c|}
\hline Negativo & Valor1 & Valor2 \\
\hline Atr.1 & 0.5 & 0.5 \\
\hline Atr.1 & 0.75 & 0.25 \\
\hline Atr.3 & 0.25 & 0.75 \\
\hline
\end{tabular}

Tabla 5 Tablas Normalizadas Fuente Elaboración Propia

Para cada tabla se elige los valores: 1 de cada atributo, es decir, la primera columna de cada tabla ver tabla 6 .

\begin{tabular}{|}
\begin{tabular}{|c|c|c|}
\hline Positivo & Valor1 & Valor2 \\
\hline Atr.1 & 0.5 & 0.5 \\
\hline Atr.1 & 0.25 & 0.75 \\
\hline Atr.3 & 0.5 & 0.5 \\
\hline \multicolumn{3}{|c|}{} \\
Negativo & Valor1 & Valor2 \\
\hline Atr.1 & 0.5 & 0.5 \\
\hline Atr.1 & 0.75 & 0.25 \\
\hline Atr.3 & 0.25 & 0.75 \\
\hline
\end{tabular}
\end{tabular}

Tabla 6 Selección de columnas Fuente Elaboración Propia

Se aplica la fórmula de teorema de Bayes y los resultados son los siguientes:

\footnotetext{
- $\quad \mathrm{P}($ positivo $\mid \mathrm{x} 5)=0,5 \cdot(0,5 \cdot 0,25 \cdot 0,5)=0,031$

25

- $\quad \mathrm{P}($ negativo $\mid \mathrm{x} 5)=0,5 \cdot(0,5 \cdot 0,75$.

$0,25)=0,046875$
}

Para la implementación del algoritmo con el software Weka, se crearon los archivos para entrenar la red bayesiana, estos archivos contienen el número de atributos utilizados con su clasificación correspondiente. Para el entrenamiento de la red bayesiana, se adjuntaron los archivos para obtener los resultados de la clasificación.

Aprendizaje_Bayesiano_Naive(ejemplos)

Para cada posible valor del resultado

Obtener estimación $\mathrm{p}^{\prime}\left(\mathrm{v}_{\mathrm{j}}\right)$ de la probabilidad $\mathrm{p}\left(\mathrm{v}_{\mathrm{j}}\right)$

Para cada valor de $\mathrm{a}_{\mathrm{i}}$ de cada atributo a

Obtener estimación $\mathrm{p}^{\prime}\left(\mathrm{a}{\sqrt{\mathrm{v}_{\mathrm{j}}}}_{\mathrm{j}}\right)$ de la probabilidad $\mathrm{p}^{\prime}\left(\mathrm{a} \sqrt{ }_{\mathrm{j}}\right)$

clasificar ejemplo $(\mathrm{x})$

$$
v_{n b=\arg \max P^{\prime}(v j) \pi(a i \mid v j)}
$$

\section{Resultados}

Para el desarrollo se utilizó un sistema Web que almacena los síntomas del paciente para posteriormente, indicar el tipo de urgencia de acuerdo a clasificación triage del sistema CAST y el posible diagnóstico que llegará a tener el paciente, sirviendo de apoyo para el médico y pueda ser atendido el paciente Figura 2.

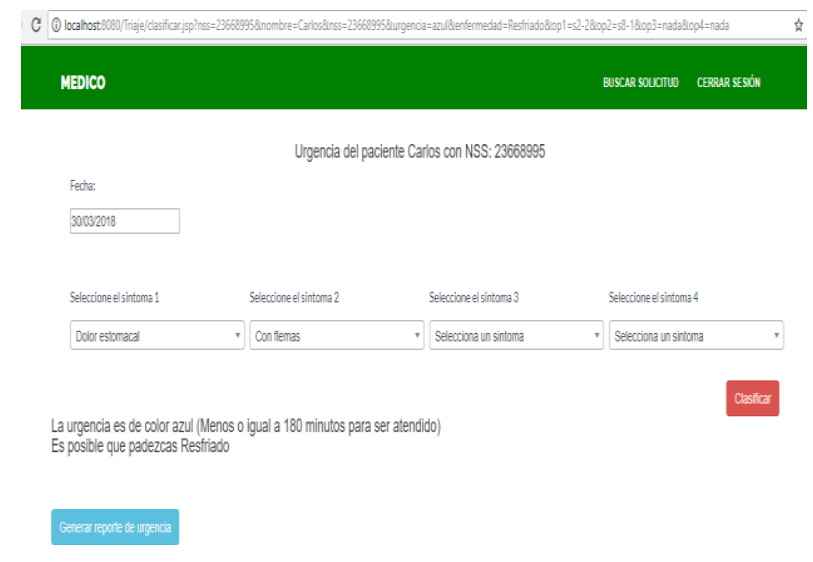

Figura 2 Clasificador de emergencias del paciente Fuente: Elaboración Propia

$\mathrm{Al}$ ser depurados los datos, se utilizaron 200 datos, los cuales se obtuvo el $80 \%$ para el entrenamiento de la red, y el $20 \%$ para pruebas. Utilizando instancias clasificadas, para la fase de aprendizaje de las probabilidades calculadas de la enfermedad y triage figura 3 . 


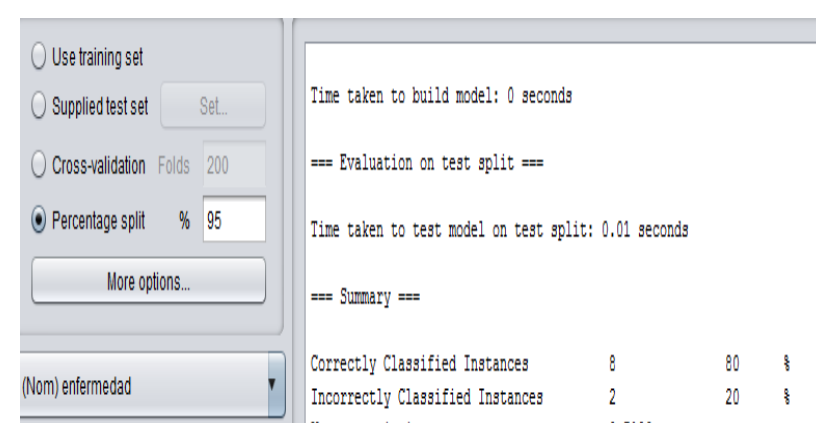

Figura 3 Instancias correctas e incorrectas de la enfermedad

Fuente: Elaboración Propia

Por consiguiente, en la aplicación móvil se realiza el registro de los síntomas con la finalidad de enviar los datos antes de ingresar a la unidad médica, datos almacenados en el sistema web, con la intención de agilizar el ingreso al hospital, y ser canalizado al área correspondiente Figura 4.

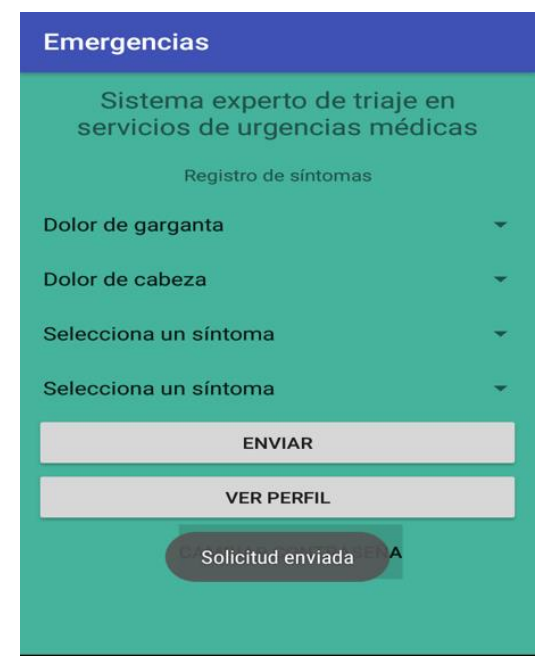

Figura 4 Formulario para envío de síntomas en aplicación móvil

Fuente: Elaboración Propia

Los resultados figura 5 indican la probabilidad de la enfermedad del paciente, datos recopilados de la aplicación móvil, donde previamente se registra el alta de urgencia del paciente como herramienta de apoyo para el personal médico. En la figura 6 se muestra para cada uno de las clasificaciones del triage de cada urgencia.

$0,1,1,-1,-1,-1,-1,2,-1,-1,-1,-1,-1,-1,-1,-1,-1,-1,-1,-1,-1,-1,-1,-1,-1$, Resfriado $-1,-1,-1,-1,-1,-1,-1,-1,-1,-1,-1,-1,-1,-1,-1,-1,0,-1,-1,-1,-1,-1,-1,-1,-1$, No_ aplica $-1,-1,-1,-1,-1,-1,-1,-1,-1,-1,-1,-1,2,-1,-1,-1,-1,-1,1,-1,-1,-1,-1,-1,-1$, No _aplica $-1,-1,-1,-1,-1,0,-1,-1,-1,-1,-1,-1,-1,-1,-1,-1,-1,-1,-1,-1,-1,-1,-1,-1,-1$, No _aplica

Figura 5 Datos utilizados para el diagnóstico de la enfermedad

Fuente: Elaboración Propia
$0,1,1,-1,-1,-1,-1,2,-1,-1,-1,-1,-1,-1,-1,-1,-1,-1,-1,-1,-1,-1,-1,-1,-1$, azul $-1,-1,-1,-1,-1,-1,-1,-1,-1,-1,-1,-1,-1,-1,-1,-1,0,-1,-1,-1,-1,-1,-1,-1,-1$, rojo $-1,-1,-1,-1,-1,-1,-1,-1,-1,-1,-1,-1,2,-1,-1,-1,-1,-1,1,-1,-1,-1,-1,-1,-1$, rojo $-1,-1,-1,-1,-1,0,-1,-1,-1,-1,-1,-1,-1,-1,-1,-1,-1,-1,-1,-1,-1,-1,-1,-1,-1$, verde $-1,-1,-1,-1,-1,-1,-1,-1,-1,-1,-1,-1,2,-1,-1,-1,-1,-1,1,-1,-1,-1,-1,-1,-1$, rojo

Figura 6 Datos utilizados para clasificación de urgencia Fuente: Elaboración Propia

El Sistema web emite reportes de los datos que son obtenidos de la aplicación móvil visualizando un diagnóstico de la posible enfermedad que el paciente pueda tener, además de clasificar el tipo de urgencia sirviendo como apoyo al médico para realizar las acciones necesarias Figura 7.

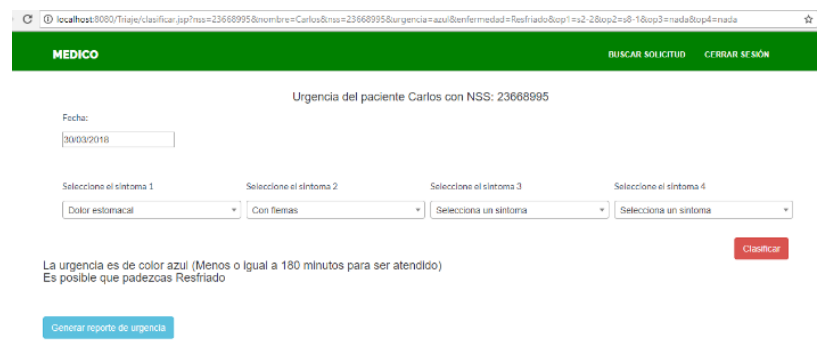

Figura 7 Diagnóstico de posible enfermedad Fuente Elaboración Propia

El reporte es visualizado en PDF, para fines que el médico requiera realizar consulta con algún doctor externo, poder enviar el diagnóstico posible. Figura 8.

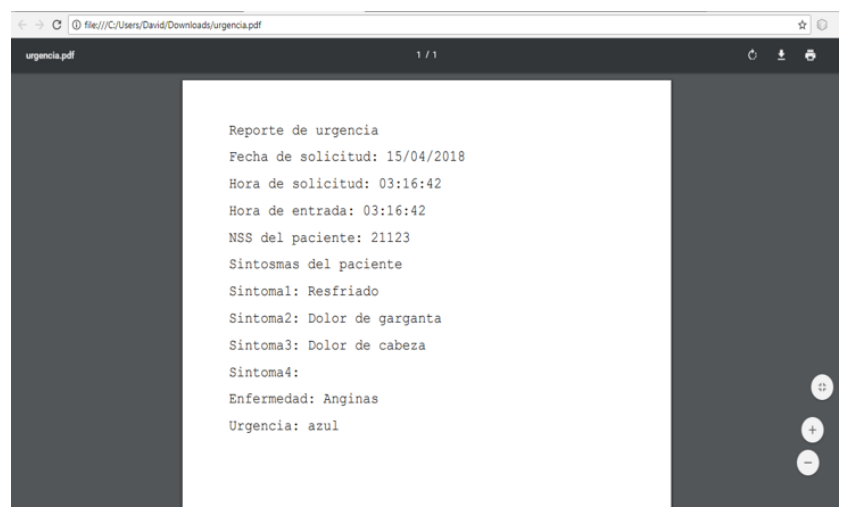

Figura 8 Generación de reporte de acuerdo a clasificación de emergencia

Fuente: Elaboración Propia

Los 200 datos que fueron utilizados para la clasificación donde previamente se realizó la limpieza de los mismos, en la gráfica 1 se visualiza el número de pacientes con la probabilidad de la enfermedad clasificada, donde se observa que la más común es infección estomacal. 
Clasificación de enfermedad

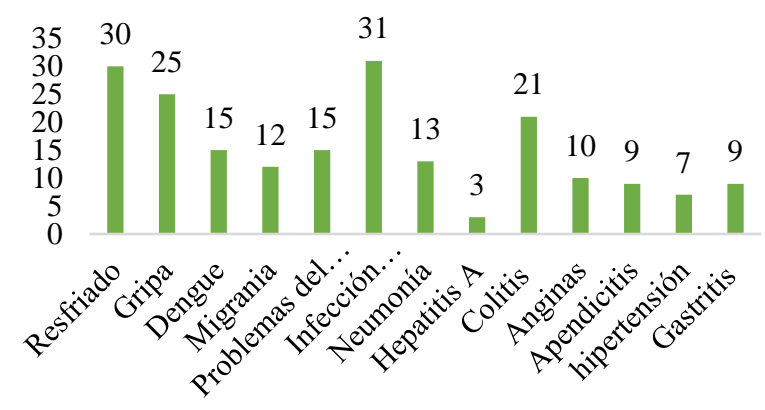

Gráfica 1 Clasificación de la enfermedad Fuente: Elaboración Propia

En la gráfica 2 se muestran la clasificación de la urgencia de cada paciente de acuerdo a la enfermedad que presenta dependiendo de los síntomas indica el color y el tiempo de respuesta para ser atendido por un personal médico.

\section{Clasificación de triage}

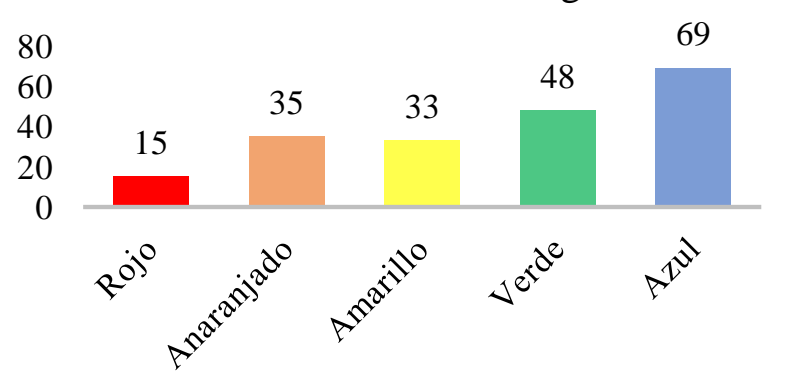

Gráfica 2 Clasificación de la urgencia Fuente: Elaboración Propia

\section{Conclusiones}

Se concluye que el sistema web con la aplicación móvil, es una herramienta de apoyo para el doctor, permitiendo al paciente registrar sus síntomas antes de ingresar a los servicios de urgencias, al utilizar el teorema de bayes se obtiene la probabilidad de clasificar la enfermedad y el nivel de urgencia que presenta el paciente.

El sistema de triage es una de las áreas de hospitales más transitadas, por lo tanto, el sistema se enfoca en contribuir en realizar un posible diagnóstico del paciente que sirva como sustento para el personal médico, permitiendo tener los datos de los síntomas del paciente que previamente registra, por lo tanto, con la información que es recolectada, es procesada mediante el teorema de bayes la posible enfermedad y el tiempo de respuesta al ser atendido el paciente con la finalidad de tener una oportuna atención médica.
La aplicación móvil brinda al paciente la oportunidad de registrar los síntomas, de tal forma que agilice el proceso de la recolección de datos, que son enviados al sistema que serán procesados con la probabilidad de realizar un diagnóstico de la posible enfermedad, ofreciendo al médico un panorama de la situación del paciente.

Como trabajo futuro, es desarrollar un módulo para el seguimiento del paciente, al ser internado llevar el tratamiento, estudios que se realicen para el control del mismo. Generando gráficas que permitan observar la evolución del paciente y generar el Alta cuando tenga que irse a casa.

\section{Referencias}

Cantón, S. F. (2006). El IMSS en cifras. La demanda de servicios en urgencias, 2004. Medigraphic , 261- 273.

Abad-Grau, M. M., Ierache, J., \& Cervino, C. (2008). Modelado de Sistema Experto para Triaje en Servicios de Urgencias Médicas. Journal of Biomedical Informatics , 432-441.

Arcos, C. R. (2017). El Triaje como herramienta fundamental para la labor de enfermería en Urgencias. (E. Group, Productor) Obtenido de Esalud https://www.ehcos.com/compartiendoexperiencias-con-los-profesionales-deurgencias-en-la-i-jornada-nacional-de-triaje/

Benavente, R. A. (2014). Sistema de Triaje en urgencias Generales. Universidad Internacional de Andalucía .

Fuente, C. D. (2006). Dificultades en la resolución de problemas que involucran el Teorema de Bayes. Un estudio exploratorio en estudiantes de psicología. Educación Matemática , 18 (2), 75 - 94.

Hernández, N. A., \& León, M. G. (2012). Satisfacción de los usuarios de urgencias basada en la sistematización del servicio. Hospital General Dolores Higalgo. Guanajuato, México. Medigraphic , 4 (1), 13-19.

J., H., M., R., \& C., F. (2004). Introducción a la Minería de Datos. Pearson, México . 
MA, V.-G., AE, M.-H., I, F.-C., \& A, B.-Y. (2017). Correlación clínica del triage con el diagnóstico clínico de ingreso y egreso realizado en los pacientes que acuden al servicio médico de urgencias de un hospital privado. Med Int Méx , 466- 475.

Morin, J. P., \& Cervantes, M. d. (2018). Comparación de métodos predictivos de minería de datos en la determinación de factores del rendimiento escolar. Revista de Sistemas Computacionales y TIC's , 4 (14), 1-9.

Páez, L. O., Lozano, M. R., \& Davila, J. A. (2011). Descripción general de la Inferencia Bayesiana y sus aplicaciones en los procesos de gestión. La simulación al servicio de la academia (2).

R, R.-R., R, T.-F., A, S.-S., \& A, A.-R. (2012). Sistema móvil de teleasistencia médica para la atención en tiempo real de casos de urgencia. Ingeniería. Investigación y Tecnología ISSN: 1405-7743, XIII (1), 1-8.

Regalado, R. F. (2009). El teorema de Bayes y su utilización en la interpretación de las pruebas diagnósticas en el laboratorio clínico. Revista Cubana de Investigaciones Biomédicas , 28 (3), 158-165.

Seixas, F. L., Zadrozny, B., Laks, J., Conci, A., \& Saade, D. C. (2014). A Bayesian network decision model for supporting the diagnosis of dementia, Alzheimer's disease and mild cognitive impairment. Computers in Biology and Medicine (51), 140 - 158.

Timarán-Pereira, S. R., Hernández-Arteaga, I., Caicedo-Zambrano, S. J., Hidalgo-Troya, A., \& Alvarado- Pérez, J. C. (2016). El proceso de descubrimiento de conocimiento en bases de datos. The Process of Knowledge Discovery on Databases . 63-86.

Torra, A. E. (2017). Sistemas de Ayuda a la Decisión Clínica en Enfermedades de Diagnóstico Complejo. 104. 